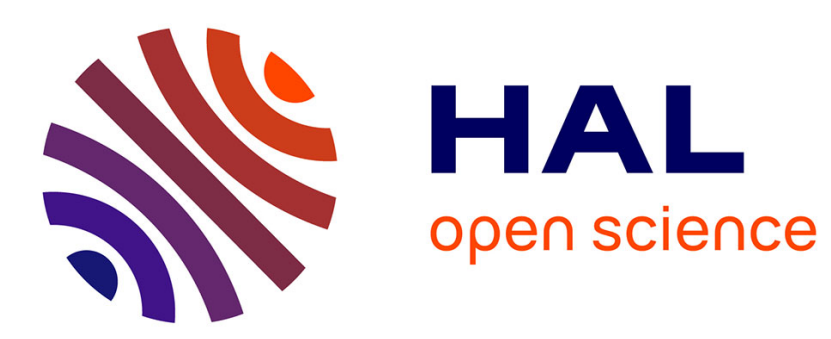

\title{
Le partage des connaissances dans les réseaux scientifiques: l'invention de règles de "bonne conduite" par les chercheurs
}

Maurice Cassier

\section{- To cite this version:}

Maurice Cassier. Le partage des connaissances dans les réseaux scientifiques: l'invention de règles de "bonne conduite" par les chercheurs. Revue française de sociologie, 1998, 39 (4), 10.2307/3323007. halshs-01894187

\section{HAL Id: halshs-01894187 \\ https://shs.hal.science/halshs-01894187}

Submitted on 12 Oct 2018

HAL is a multi-disciplinary open access archive for the deposit and dissemination of scientific research documents, whether they are published or not. The documents may come from teaching and research institutions in France or abroad, or from public or private research centers.
L'archive ouverte pluridisciplinaire $\mathbf{H A L}$, est destinée au dépôt et à la diffusion de documents scientifiques de niveau recherche, publiés ou non, émanant des établissements d'enseignement et de recherche français ou étrangers, des laboratoires publics ou privés. 


\section{Le partage des connaissances dans les réseaux scientifiques:} l'invention de règles de "bonne conduite " par les chercheurs Maurice Cassier

\section{Citer ce document / Cite this document :}

Cassier Maurice. Le partage des connaissances dans les réseaux scientifiques : l'invention de règles de « bonne conduite " par les chercheurs. In: Revue française de sociologie, 1998, 39-4. pp. 701-720;

doi : $10.2307 / 3323007$

https://www.persee.fr/doc/rfsoc_0035-2969_1998_num_39_4_4838

Fichier pdf généré le 23/04/2018 


\section{Resumen}

Maurice Cassier : La partitción de conocimientos en las redes científicas : la invención de las reglas de « buena conducta » por los investigadores.

Las nuevas formas de invención colectiva, redes o consorcios de investigación, solicitan la invención de reglas de gestión de los conocimientos que sean susceptibles de conciliar la distribución de los mismos con un cierto grado de protection individual, o mejor el derecho de publicación de los investigadores universitarios y la de reserva de los resultados solicitados por las firmas industriales. Los investigadores del proyecto Bridge sobre los lipases han imaginado un dispositivo ingenioso para difundir los datos que permitan combinar un derecho reservado temporal para el propietario de los datos, su distribución en el espacio colectivo del proyecto, y su publicación relativamente rápida. Estas reglas de juego, elaboradas por los investigadores, lejos de los servicios jurídicos, son susceptibles de circular entre las redes científicas. Estos consortium agreements hacen aparecer nuevas reglas, sino nuevos derechos, de gestión de los conocimientos. reservado temporal para el propietario de los datos, su distribución en el espacio colectivo del proyecto, y su publicación relativamente rápida. Estas reglas de juego, elaboradas por los investigadores, lejos de los servicios jurídicos, son susceptibles de circular entre las redes científicas. Estos consortium agreements hacen aparecer nuevas reglas, sino nuevos derechos, de gestión de los conocimientos.

\section{Zusammenfassung}

Maurice Cassier : Die Wissensteilung in den wissenschaftlichen Netzen : die Erfindung der « Anstandsregeln » durch die Forscher.

Die neuen kollektiven Erfindungsformen (Forschungsnetze oder Konsortia) verlangen die Erfindung von Führungsregeln des Wissens, die sowohl die Wissensteilung als auch einen gewissen Grad von Eigenschutz vereinbaren, oder auch das Recht zu Veröffentlichung der Hochschulforscher und das Ergebnis-Reservierungsrecht, wie es von Industriefirmen gefordert wird. Die Forscher des Projekts Bridge zu den Lipasen, haben ein kluges Verteilungssystem der Daten ausgedacht, mit dem ein zeitweises Reservierungsrecht für den Dateninhaber, die Datenbeteiligung in dem kollektiven Raum des Projekts und die relativ schnelle Veröffentlichung kombiniert werden. Diese von den Forschern unabhängig von Rechtsabteilungen erarbeiteten Spielregeln, könnten für die Wissenschaftsnetze gültig werden. Diese consortium agreements führen zu neuen Regeln oder sogar neuen Rechtsgrundlagen für die Wissensführung. der Daten ausgedacht, mit dem ein zeitweises Reservierungsrecht für den Dateninhaber, die Datenbeteiligung in dem kollektiven Raum des Projekts und die relativ schnelle Veröffentlichung kombiniert werden. Diese von den Forschern unabhängig von Rechtsabteilungen erarbeiteten Spielregeln, könnten für die Wissenschaftsnetze gültig werden. Diese consortium agreements führen zu neuen Regeln oder sogar neuen Rechtsgrundlagen für die Wissensführung.

\footnotetext{
Abstract

Maurice Cassier : Sharing knowledge in the scientific network : inventing rules for « good conduct » among researchers.

Due to new forms of collective invention, research networks or consortia, new rules are required to manage this knowledge, allowing for both a mutual sharing of knowledge and at the same time a certain degree of individual protection, or the right for university researchers to publish material, while keeping a reservation on results asked for by industrial firms. Researchers working on the Bridge lipase project came up with an ingenious way to diffuse data combining, at the same time, the possibilities to maintain the temporary reservation of rights for the data owner, to share the data within the collective sphere of the project and lastly to allow a relatively rapid publication of the data. This set of rules, developed by the researchers without any help from a legal department, will most likely be followed on scientific networks. These agreement consortia are bringing to light new rules, if not new rights, as regards knowledge management. project and lastly to allow a relatively rapid publication of the data. This set of rules, developed by the researchers without any help from a legal department, will most likely be followed on scientific networks. These agreement consortia are bringing to light new
} 
rules, if not new rights, as regards knowledge management.

\section{Résumé}

Les nouvelles formes d'invention collective, réseaux ou consortia de recherche, demandent l'invention de règles de gestion des connaissances susceptibles de concilier le partage des connaissances et un certain degré de protection individuelle, ou encore le droit de publication des chercheurs universitaires et celui de réservation des résultats demandé par les firmes industrielles. Les chercheurs du projet Bridge sur les lipases ont imaginé un dispositif ingénieux de diffusion des données, qui permet de combiner un droit de réservation temporaire pour le propriétaire des données, leur partage dans l'espace collectif du projet, et leur publication relativement rapide. Ces jeux de règles, élaborés par les chercheurs à l'écart des services juridiques, sont susceptibles de circuler entre les réseaux scientifiques. Ces "consortium agreement" font émerger de nouvelles règles, sinon de nouveaux droits, de gestion des connaissances. 
Maurice CASSIER

\title{
Le partage des connaissances dans les réseaux scientifiques : l'invention de règles de «bonne conduite» par les chercheurs
}

\begin{abstract}
RÉSUMÉ
Les nouvelles formes d'invention collective, réseaux ou consortia de recherche, demandent l'invention de règles de gestion des connaissances susceptibles de concilier le partage des connaissances et un certain degré de protection individuelle, ou encore le droit de publication des chercheurs universitaires et celui de réservation des résultats demandé par les firmes industrielles. Les chercheurs du projet Bridge sur les lipases ont imaginé un dispositif ingénieux de diffusion des données, qui permet de combiner un droit de réservation temporaire pour le propriétaire des données, leur partage dans l'espace collectif du projet, et leur publication relativement rapide. Ces jeux de règles, élaborés par les chercheurs à l'écart des services juridiques, sont susceptibles de circuler entre les réseaux scientifiques. Ces consortium agreement font émerger de nouvelles règles, sinon de nouveaux droits, de gestion des connaissances.
\end{abstract}

La prolifération des formes d'invention collective, dans le cadre de consortia ou de réseaux de recherche composés de laboratoires publics, industriels ou associant les deux, demande l'invention de nouvelles règles d'attribution et de circulation des données des recherches réalisées en collaboration, en complément du droit de la propriété intellectuelle existant. Nous faisons l'hypothèse que les réseaux d'alliances hétérogènes (Callon et al., 1995) sont des lieux de forte créativité en matière de gestion de la propriété intellectuelle et du droit d'accès aux connaissances. Les difficultés à résoudre sont multiples. Il s'agit, en effet, de concevoir des contrats ou des règles communément admises par les participants qui, d'un côté, stimulent le partage des données et la création collective, et, d'un autre, garantissent un certain degré de protection individuelle. Il s'agit encore de concilier la priorité de publication qui motive les chercheurs académiques et un droit de réservation des résultats demandé par les industriels. Il s'agit enfin, en raison de l'intégration grandissante de la science et du marché, de gérer des situations hybrides dès lors que l'on recourt à deux modes de jugement des résultats de l'activité de recherche, par les pairs et par le marché. 
Ce besoin de régulation en matière de propriété et de droit d'accès aux données de la recherche est souligné tant par les économistes que par les sociologues de l'innovation. Dans ses travaux sur la montée des réseaux technico-économiques, M. Callon (1992) fait l'hypothèse que «l'organisation économique, comme mode de coordination d'activités diversifiées mais complémentaires, s'étend au-delà de la sphère industrielle et du seul monde des entreprises. Les centres de recherche publics ou semi-publics, les centres techniques, les bureaux d'études et d'ingénierie deviennent des acteurs économiques à part entière, de même que les pouvoirs publics [...] Entre directement dans le jeu économique toute une série d'acteurs qui viennent en bouleverser les règles [...] Un régime nouveau se met en place qui se caractérise par des formes originales d'interactions, de régulation et des contenus d'activités qui sont eux-mêmes inédits ». Dans la réflexion qu'ils conduisent sur les nouveaux faits stylisés de l'appropriabilité de la recherche, Cohendet, Héraud et Zuscovitch (1992) soulignent l'importance qu'il y a à réfléchir sur les règles de partage dans les accords de coopération: «Ce qui réclame le plus d'imagination et d'innovation juridique, c'est la gestion des coopérations [...] Si la forme réseau devient la norme de l'innovation technologique, la figure dominante du nouveau système industriel, alors il faudra innover de manière plus radicale en matière de forme juridique de l'association économique, afin d'établir un mode d'appropriation des activités innovantes mieux adapté, et donc plus incitatif pour le progrès technique global.» Nous entendons développer ce programme de recherche en étudiant l'invention de règles de bonne conduite écrites par les participants à un consortium de recherche européen en biotechnologie.

Le projet étudié, le programme Bridge sur les enzymes lipasiques d'intérêt industriel (1), mobilise un réseau d'acteurs particulièrement hétérogènes puisqu'il regroupe cinq grandes firmes industrielles largement concurrentes entre elles, une société de biotechnologie créée par des chercheurs et seize laboratoires appartenant à des universités ou des organismes de recherche publics. La gestion de la coopération implique des négociations de tous ordres et de multiples compromis. Il faut parvenir à concilier les intérêts divergents des industriels qui participent au projet et leur garantir un minimum de protection afin qu'ils acceptent de s'engager dans la coopération: «On va quand même échanger de l'information un peu privée, c'est ça l'idée. » [Scientific officer de la CEE]. Il faut ensuite concilier un certain degré d'appropriation des connaissances et le droit de publication des chercheurs académiques. Il faut enfin organiser un pool de connaissances partagé par tous les membres du réseau.

(1) Les enzymes lipasiques sont des protéines qui dégradent les corps gras. Comme telles, elles intéressent 1 'industrie des déter- gents, les industries agro-alimentaires, la chimie fine et l'industrie pharmaceutique. 
La description aussi fine que possible des arrangements négociés par les acteurs du consortium sur les lipases (2) nous permettra d'avancer sur la gestion des connaissances dans un contexte d'invention collective et sur la capacité de régulation économique et juridique des acteurs locaux, en l'occurence des chercheurs.

Concernant la gestion des connaissances dans des réseaux d'innovation, la moisson est riche puisque les guidelines du projet Lipases instaurent en premier lieu un ingénieux mécanisme de diffusion concentrique des données qui permet de conjuguer, dans le temps, la réservation des résultats dans le cadre d'une équipe restreinte, le partage des données dans l'espace collectif du réseau, puis leur publication rapide. On voit en second lieu émerger de nouvelles catégories pour gérer la propriété intellectuelle, comme celle du «droit de premier usage des connaissances» qui confère au détenteur d'une donnée un droit de réservation avant même que la connaissance ne soit inscrite dans un dispositif technique brevetable, à l'opposé du droit des brevets qui exige que l'inventeur soit en mesure de décrire une application industrielle. Les juristes y voient une nouveauté absolue (Hermitte, 1995). Nous pensons également que les consortia de recherche font émerger une nouvelle catégorie de biens, «les données collectives» ou «données de réseau», qui présentent la particularité de conjuguer des aspects des biens publics et des biens privés. Ce sont des données partagées dans un réseau afin de stimuler l'invention collective, le temps de la recherche. Si elles sont partagées par les participants, elles excluent les non-membres. Elles sont, selon nous, caractéristiques du développement des nouveaux collectifs de recherche entre des institutions hétérogènes.

Concernant le processus de régulation, les enseignements du projet Lipases n'en sont pas moins riches. 11 fait apparaître la capacité de régulation des chercheurs qui s'affirment comme des acteurs économiques à part entière. Ce sont eux qui ont élaboré «les règles de bonne conduite » du projet Lipases, à l'écart des services juridiques des firmes ou des organismes publics de recherche engagés dans le projet. Il souligne l'importance des régulations locales en matière de propriété intellectuelle, en complément des dispositifs contractuels ou juridiques plus généraux (en

(2) Nous avons suivi les discussions entre les chercheurs, le coordinateur scientifique, le coordinateur administratif de la Commission européenne et les firmes, pour la mise au point des Rules and guidelines du projet Lipases entre 1990 et 1992. Nous avons recueilli le code de bonne conduite du projet voisin sur le séquençage du chromosome de la levure intitulé «The perfect gentleman sequencer», qui a inspiré les Rules and guidelines du projet Lipases. Nous avons collecté les contrats signés entre la CEE et les différentes équipes ainsi que des éléments de la négociation sur la création d'une plateforme industrielle accessible aux industriels non membres du projet. Nous avons enfin observé les échanges au sein du consortium (échanges de matériel biologique, de données sur des supports variés, de personnel, mise à disposition d'équipements rares, production de mutants spécifiques à la demande, etc) et les premiers résultats obtenus (publications individuelles ou communes, bases de données, enzymes mutantes, brevets). 
l'occurence les contrats types de la CEE et le droit de la propriété industrielle). Il démontre l'existence d'une pluralité de normes locales de gestion des connaissances, incluant une grande variété de compromis entre le privé et le public, ce qui réduit l'hypothèse de l'omniprésence des normes mertoniennes de la «science ouverte», détachées des intérêts économiques et des applications industrielles.

\section{La formation d'un réseau d'alliances hétérogènes : coordination sociotechnique et dispositifs de régulation des échanges}

Ce réseau de laboratoires publics et privés, fédéré par les pouvoirs publics européens, vise à accélérer la production de connaissances de base sur des matériels biologiques d'intérêt industriel. Les partenaires se sont accordés pour sélectionner un échantillon de dix à quinze enzymes lipasiques qui ont pour propriété de dégrader les corps gras. Les connaissances produites auront une double valeur d'usage : académique, dans la mesure où la famille des lipases est moins connue que d'autres familles d'enzymes, et industrielle, par le fait que les connaissances obtenues sur la structure et l'activité cinétique de ces protéines seront utilisées pour améliorer leur utilisation industrielle, pour concevoir de nouveaux produits dans l'industrie des détergents, l'industrie pharmaceutique et l'industrie agro-alimentaire, ou encore pour imaginer des protéines mutantes plus efficaces. La demande industrielle stimule le développement de la recherche de base en biochimie. La Commission européenne entend, pour sa part, renforcer un pôle compétitif de la CEE (3), en intensifiant les transferts de connaissances entre les laboratoires publics et les firmes, grandes et petites : «L'industrie européenne pourra bénéficier d'un accès direct et privilégié aux résultats de la recherche fondamentale sur cette famille d'enzymes qui est d'un intérêt stratégique. » [Document préparatoire, CEE, 1989].

Ce consortium mixte, universitaire et industriel, ne part pas de rien. Il s'appuie sur des tronçons de réseaux préexistants, spontanés ou organisés par les pouvoirs publics. Il existe des relations, parfois anciennes, entre les laboratoires publics et les laboratoires industriels dans le domaine de

(3) Pour reprendre la terminologie du Centre de sociologie de l'innovation, les deux pôles, science et marché, sont bien réprésentés en Europe dans ce domaine. La Recherche et développement européenne sur les lipases bénéficie d'une avance sur son homologue américaine tandis que le Japon est un compétiteur sérieux au niveau des applications (Arguments pour un projet $T$ sur la caractérisation multidisciplinaire des lipases présentant un intérêt pour des applications industrielles, Document CEE, 1989). En ce qui concerne le marché, les firmes européennes productrices d'enzymes, principalement néerlandaises et danoises, contrôlent $80 \%$ du marché mondial. L'établissement, ou plutôt le resserrement des liens entre les pôles science et marché sont donc jugés stratégiques par la division des Biotechnologies de la Commission européenne. 
la recherche sur les lipases (4). Plus récemment, au cours des années 1980 , les contrats de recherche se sont multipliés entre laboratoires publics et firmes. Des publications communes, des échanges de chercheurs, des prototypes commercialisés témoignent du travail réalisé ensemble, et ceci à l'échelle européenne. Les pouvoirs publics avaient déjà encouragé des regroupements : en 1985, la CEE finance une collaboration de sept laboratoires publics; en 1986, le programme Biotechnologie du ministère de la Recherche finance un projet coopératif qui associe vingt laboratoires universitaires et industriels. Toujours en France, un réseau spontané et informel, regroupant des chercheurs publics et des firmes pharmaceutiques, a perduré au projet du ministère de la Recherche, à la fin des années 1980 . Les participants à ce réseau souhaitaient préserver un espace de circulation des connaissances sur les lipases qui soit indépendant, sinon concurrent, des grandes sociétés de biotechnologies européennes : «On essaye de mettre en place un pool Lipases depuis le fondamental jusqu'au pré-appliqué, de faire en sorte que l'information circule quand même.» [Professeur de biotechnologie, Compiègne].

Le réseau Lipases s'appuie sur des liens de complémentarité, de similarités et de dépendances réciproques entre les acteurs publics et privés. Il existe une forte complémentarité entre les laboratoires publics et les firmes industrielles dans ce domaine : les coordinateurs du projet Lipases attendent des firmes qu'elles mettent leurs souches industrielles à la disposition du projet, ce qui permettra d'obtenir des connaissances inédites ou de produire des enzymes mutantes en croisant les souches des uns et des autres. Il sera également possible d'associer des disciplines différentes, la biochimie du laboratoire du CNRS de Marseille et la biologie moléculaire d'une grande firme de biotechnologie danoise pour produire des mutants, ou encore de combiner des phases différentes de l'activité de Recherche et développement, la recherche de base sur la cinétique des enzymes réalisée dans des milieux modèles et les tests en milieux réels réalisés dans l'industrie.

Il existe également, dans le domaine des lipases, une relative similarité des compétences des laboratoires académiques et des laboratoires industriels : «Un bon nombre d'excellents laboratoires européens, tant académiques qu'industriels, sont engagés dans l'étude des lipases. » [Arguments pour un projet $\mathrm{T}$ ]. Les grandes firmes productrices ou utilisatrices d'enzymes hébergent dans leurs murs une forte recherche de base, parfois très ancienne (depuis les années 1940 pour la firme danoise Novo). Dans un tel cas de figure, les laboratoires industriels deviennent des alliés scienti-

(4) Le laboratoire de lipolyse enzymatique de Marseille, qui est aujourd'hui le coordinateur scientifique du projet européen, a organisé un colloque international sur les lipases au début des années 1960. Les laboratoires industriels, notamment celui d'Unilever, y étaient fortement représentés (10 chercheurs présents en 1961). Le fondateur du laboratoire, P. Desnuelle, entretenait une correspondance scientifique régulière avec plusieurs firmes de l'industrie des corps gras au cours des années 1960 et 1970 . 
fiques de premier plan : «On est dans une situation tout à fait nouvelle : si on veut continuer à être dans le coup sur les lipases, il est vital pour nous, universitaires, de participer à un tel projet pour les informations que nous allons en tirer. Il y a un tel investissement de la part des industriels qu'ils deviennent des partenaires privilégiés sur le plan de la connaissance, sur le plan scientifique.» [Responsable du Laboratoire de lipolyse enzymatique, CNRS, Marseille]. Ainsi, les deux premières publications sur la structure des lipases ont été cosignées dans la revue Nature par des universitaires et deux firmes industrielles, Novo et Hoffmann La Roche. La forte capacité de recherche interne des firmes facilite la coopération avec la recherche universitaire et elle augmente les bénéfices qu'elles pourront tirer des résultats du projet. Enfin, pour cette classe d'enzymes, les laboratoires industriels ne peuvent se passer des laboratoires publics (5). Ces derniers détiennent un stock de connaissances et de savoir-faire souvent considérables, accumulés depuis plus de trente ans. Par exemple, le Laboratoire de lipolyse enzymatique du CNRS de Marseille, qui coordonne le projet Lipases, a développé des instruments scientifiques uniques pour mesurer l'activité de cette classe d'enzymes. De fait, il est présent dans quatre des cinq sous-projets du programme Lipases.

On peut parler, pour les lipases, d'un réseau technico-économique relativement convergent (Callon, 1992), au sens où tous les acteurs impliqués, académiques et industriels, partagent un objet commun, la famille des enzymes lipasiques. Ils parlent un langage commun, publient dans les mêmes revues et se rencontrent dans les mêmes congrès. Il ne leur sera pas difficile de codéfinir le programme Bridge sur les lipases. Le rôle des pouvoirs publics se limitera ici à fédérer et à étendre les réseaux existants.

En revanche, la régulation des échanges au sein du consortium s'annonce particulièrement difficile (6).

Première difficulté, les six grandes firmes qui participent au projet sont largement concurrentes. Tout d'abord, certaines sont productrices d'enzymes, d'autres en sont utilisatrices. Chaque partie essaye de contrôler l'invention de nouvelles méthodes d'utilisation des lipases ou la création de nouvelles lipases mutées pour s'approprier la rente d'innovation. Ensuite, elles sont inégalement avancées sur le sujet. Parmi celles-ci, une grande firme danoise de biotechnologie détient une avance substantielle sur ses concurrentes, en termes de Recherche et développement aussi bien

(5) À la différence de ce que l'on observe pour d'autres familles d'enzymes où la recherche est localisée principalement dans la sphère privée : «Les protéases sont partiellement faites, la moitié au dessous de la table [...] Il y avait énormément de travail fait par l'industrie sur les protéases, mais du travail confidentiel. Ce n'était pas la peine de remettre de l'argent public pour travailler sur quelque chose qui était peut-être déjà fait, mais disons dans l'ombre, caché, pour des raisons de secret commercial.» [Scientific officer].

(6) La convergence du réseau sociotechnique tend à aiguiser les rapports de concurrence dans la mesure où tous les acteurs du réseau sont capables de dupliquer assez facilement les connaissances et les techniques mises au point. 
qu'en termes d'applications (elle commercialise déjà une lessive à base de lipases au Japon). Elle est évidemment réticente à partager de l'information, même partiellement : «Eux sont plutôt réticents à partager avec tout le monde l'information qu'ils ont accumulée pendant toute la phase de Recherche et développement antérieure au projet Bridge. On ne peut pas leur demander de tout dévoiler à leurs concurrents parce qu'il y a une action européenne! Il n'empêche qu'ils participent à l'action [...] C'est quand même étonnant de tous les retrouver dans ce projet, parce qu'à un moment, on se demandait s'ils allaient vraiment participer, parce qu'il n'était pas évident que partager des informations soit une bonne stratégie pour eux. » [Coordonnateur scientifique] (7). Enfin, les firmes participantes pouvaient être réticentes à l'idée de faire subir des tests comparatifs à leurs souches d'enzymes, au risque de découvrir de moindres performances que celles affichées par leurs rivales.

Deuxième difficulté, la barrière à l'entrée du projet est élevée pour les PME qui ne possèdent pas de capacités de recherche suffisantes pour faire une proposition complète à l'appel d'offres du programme (c'est-à-dire une proposition qui intègre l'étude de la structure et de la fonction d'une ou plusieurs enzymes) et pour se lancer durant plusieurs années dans une recherche en amont des applications. De fait, les PME qui ont participé à la phase de définition du programme ne sont pas à l'arrivée. On n'en retrouve qu'une seule dans le consortium final. Cette société de biotechnologie issue de l'université a été recrutée par un groupe de laboratoires publics qui souhaitaient préserver un espace de recherche indépendant des grandes firmes dominantes.

Troisième difficulté, le projet de création d'une plateforme industrielle, accessible aux entreprises européennes qui ne participent pas directement au projet, risque de se heurter à l'opposition des firmes membres du projet. Cette opposition entre membres et non-membres est assez répandue dans les consortia de recherche, les premiers ne souhaitant pas partager trop vite les bénéfices de la recherche collective avec les derniers (8).

Dernière difficulté, industriels et universitaires sont susceptibles de s'opposer sur les délais de publication des résultats ou sur la publication partielle de certaines données d'intérêt industriel. La publication partielle des données structurales des protéines est vivement dénoncée par des universitaires, qui se plaignent de ne pas pouvoir utiliser ces données fragmentaires pour faire des comparaisons.

Comment inciter les entreprises à s'engager dans la recherche collective, notamment en acceptant de communiquer leurs souches d'enzymes confidentielles?

(7) Certaines firmes très soucieuses de protéger leurs données, en l'occurence les laboratoires pharmaceutiques sollicités par les chercheurs français, se sont abstenues de par- ticiper au projet Lipases. Elles dénoncent les clauses de divulgation des résultats de recherche dans les contrats types de la CEE.

(8) Voir Grindley, Mowery, Silverman (1994). 
Tout d'abord, les participants se sont accordés pour remonter en amont des applications : «Si le choix des enzymes a été fait sur des critères d'application potentielle ou même existante, le financement de l'étude doit porter sur des données fondamentales qui devront être partagées [...]. On a éliminé tout ce qui était trop près du développement, trop près des applications, pour éviter toute interférence, toute possibilité de conflit d'intérêt entre industriels [...]. À mon avis, le seul moyen de faire cohabiter pendant un temps des industries concurrentes, c'est de remonter vers l'amont.» [Coordinateur scientifique]. Les entreprises gardent dans leurs murs les connaissances biotechnologiques liées à la mise en œuvre des enzymes. Elles consentent à partager des connaissances de base sur les mêmes enzymes. Cette séparation en amont et aval n'est toutefois pas absolue : ainsi les connaissances sur la structure d'une enzyme peuvent être immédiatement utilisées pour produire un mutant d'intérêt industriel, susceptible d'être protégé par un brevet. De fait, les travaux du consortium ont débouché sur un brevet.

Ensuite, le consortium a été segmenté en cinq sous-projets au sein desquels les entreprises concurrentes se répartissent (9). Ce mode d'organisation, adopté par de nombreux consortia de recherche (Aldrich, Sasaki, 1995), délimite des espaces de collaboration séparés et protégés de la concurrence. Chaque industriel peut travailler sur ses propres enzymes en collaboration avec les laboratoires publics avec lesquels il est associé, sans risque de divulgation. Si cette organisation favorise l'appropriation des données de recherche, elle complique les possibilités d'échange et de création collective. Outre la mise en place d'une structure de coordination scientifique entre les sous-projets, animée par le leader scientifique du domaine, les participants ont défini une série de compromis entre des espaces de recherche réservés et un espace de recherche collective.

\section{Les solutions de compromis entre bien privé, bien collectif et bien public : règles et pratiques d'échange}

Ces solutions de compromis entre ce qui relève du domaine privé, du domaine collectif et de l'espace public reposent sur un jeu de règles explicites élaborées par les participants, les Rules and guidelines du projet Lipases, et sur des pratiques d'échange des données et des matériels biologiques qui ont été adoptées par les membres du consortium.

(9) Cinq contrats séparés ont été signés avec cinq groupes distincts : trois d'entre eux sont pilotés par une grande société qui est associée à deux ou trois laboratoires publics; un autre projet regroupe deux firmes indus- trielles et six laboratoires publics; enfin le dernier projet, à dominante publique, rassemble huit laboratoires académiques et une PME de biotechnologie qui fournira des enzymes et fera des travaux de biologie moléculaire. 


\section{La circulation des données}

Les Rules and guidelines du projet Lipases instaurent un dispositif de diffusion concentrique des données, en fonction du temps : «Pour garantir la diffusion maximale de l'information au sein de l'ensemble du projet, plusieurs périodes ont été définies pour la distribution des données structurales et fonctionnelles obtenues sur les lipases étudiées. »[Article 7]. Ce dispositif prévoit quatre degrés d'accessibilité ou de publicité des résultats de recherche : premier cercle, celui des données d'équipes ou team data, qui sont accessibles aux seuls membres d'un sous-projet, c'est-à-dire un industriel et quelques laboratoires publics. Elles doivent être transférées dès que possible dans l'espace collectif des vingt-deux participants, au plus tard six mois après leur entrée dans le sous-projet; second cercle, celui des données collectives ou pooled data, qui sont accessibles à tous les membres du consortium. Les participants ne doivent pas les communiquer à des tiers sans l'autorisation du propriétaire des données. Les Guidelines fixent un délai de 6 mois pour leur publication; troisième cercle, celui de la plateforme industrielle: les données sont diffusées aux membres de la plateforme industrielle, qui regroupe des industriels utilisateurs qui ne ne font pas partie du consortium et qui acquittent un droit d'accès pour bénéficier d'une information avant publication; quatrième cercle, l'espace public. Les public data sont accessibles à tous les utilisateurs potentiels, via des publications scientifiques ou le dépôt des résultats dans une base de données publique.

Le point central de l'accord porte sur le délai de rétention et de diffusion des données. Les universitaires ont intérêt à accélérer le passage des données dans le domaine public, tandis que les industriels peuvent souhaiter retenir les données plus longtemps, notamment en cas de dépôt d'un brevet.

À la fín de l'année 1990 , soit un an après le démarrage du projet, le laboratoire du CNRS de Marseille, dont le responsable est le coordinateur scientifique de l'ensemble du projet, proposa de réduire les périodes de rétention des données au sein des équipes et dans le consortium à trois mois, le délai de publication ne devant pas dépasser six mois: «Il y a des étudiants en thèse qui ne peuvent rester un an sans publier.» Cette proposition fut appliquée, bien que réduisant de moitié le délai de publication prévu dans les Guidelines. Elle ne concernait toutefois qu'une partie des données, les données cinétiques, qui étaient produites uniquement par le laboratoire du CNRS de Marseille. Celui-ci recevait les échantillons d'enzymes de tous les participants. Il les étudiait sur les instruments de mesure spécifiques en sa possession. Au terme de la recherche, les enzymes étaient restituées à leur propriétaire en même temps que les données obtenues. Celui-ci disposait alors de trois mois pour les évaluer et prendre des mesures de protection. Au bout de ces trois mois, le laboratoire producteur des données les diffusait de façon synchrone à tous les partenaires du 
consortium et au bout de six mois, les données étaient publiées. Le raccourcissement du délai de publication fut rendu possible par le caractère «amont» des données et ne fut accepté que grâce à l'influence particulière du laboratoire marseillais dans le consortium. Il était le seul à posséder certaines techniques et son patron jouissait d'une réputation particulière au sein de la communauté scientifique spécialiste des lipases. De plus, il était capable d'exercer un contrôle plus serré sur la diffusion des données et les flux d'échantillons dans la mesure où il était le seul producteur du consortium. La gestion des données structurales était beaucoup plus complexe à organiser du fait qu'il existait cinq producteurs répartis dans les cinq sous-projets. Elle était aussi plus sensible, en raison de la concurrence entre les firmes participantes (un défaut de coordination, par exemple la diffusion non synchrone des données, pouvait se traduire par un avantage pour un groupe au détriment d'un autre).

Si les règles ont été modulées de façon à réduire le délai de publication, elles autorisaient aussi l'allongement du délai de réservation, si un participant le demandait (10). La préparation d'une demande de brevet pouvait nécessiter un surcroît de rétention des données.

Ce dispositif introduit plusieurs nouveautés en matière d'appropriation et de partage des résultats de recherche. Il crée tout d'abord un droit de réservation temporaire sur des connaissances qui ne sont pas brevetables en l'état, ce qui donne du temps au détenteur des données pour évaluer leur valeur d'usage industrielle et pour décider ou non de faire des développements pour les protéger. Ce droit de premier usage des connaissances est très différent de la règle du brevet qui ne peut s'appliquer qu'à des inventions inscrites dans des dispositifs techniques sufissamment avancés pour être décrits et reproduits par des tiers. Il est parfaitement adapté à la protection de connaissances de base qui sont susceptibles d'avoir une valeur industrielle ou commerciale. C'est le cas de nombreuses connaissances en biotechnologie, à l'instar des données structurales des protéines qui, en l'état, peuvent être vendues à des laboratoires pharmaceutiques pour être utilisées dans la conception de nouveaux médicaments. De même, les données fonctionnelles produites par le consortium européen intéressent immédiatement la recherche industrielle sur les détergents pour améliorer ses lessives. Ce droit de premier usage des connaissances est formulé dans le contexte d'une intégration croissante de la science, de l'économie et du développement d'une recherche de base qui produit à la fois pour l'académie et pour l'industrie (Cassier, 1996). Les données fonctionnelles sur les lipases donneront lieu à des publications internationales, dont une

(10) «Dans des situations exceptionnelles, le coordinateur de l'équipe pourra requérir l'autorisation de retenir les données pendant un délai plus long que les périodes indiquées. Une telle requête devrait être dis- cutée avec et accordée par le scientific officer de la Commission des Communautés européennes qui est responsable des contrats. » [Rules and guidelines, article 10]. 
dans Nature, et à des applications industrielles qui seront développées dans les murs des firmes participantes.

La délimitation d'un espace réservé pendant quelques mois est d'un grand intérêt pour les firmes industrielles qui se sont engagées dans le consortium. Elles sont ainsi en mesure de mieux contrôler la circulation de leurs matériels et des données produites par le groupe. Ce délai leur confère une avance sur leurs concurrents, d'autant que seules les données publiables sont divulguées, et non les matériels biologiques et les savoirfaire de laboratoire utilisés pour les obtenir. Ce dispositif, initialement conçu dans le cadre d'un consortium purement universitaire, le réseau européen sur la levure, afin de récompenser les laboratoires en leur attribuant la propriété temporaire des données qu'ils avaient obtenues, a été remobilisé par le consortium sur les lipases qui devait réussir à faire coexister des firmes concurrentes. La force du dispositif est d'introduire un certain degré de protection individuelle dans un espace de recherche collective.

La seconde innovation des Rules and guidelines est de définir la catégorie de «données collectives» (pooled data). Il s'agit des données qui sont partagées entre tous les membres du consortium. C'est l'existence des pooled data qui détermine les possibilités d'invention collective du réseau. L'accès à une connaissance relative à une protéine $\mathrm{A}$ permet en effet d'avancer plus rapidement dans la caractérisation d'une protéine $B$. Les membres du réseau peuvent exploiter les interdépendances existant entre les objets de recherche, qui appartiennent ici à une même famille biologique. Ils sont en mesure d'accéder à l'ensemble de la collection des données produites par le consortium, ce qui autorise des comparaisons et de nouvelles synthèses. Le partage des connaissances produit un effet économique particulier - l'effet de collection - qui renvoie à la possibilité d'obtenir un surplus de connaissances grâce à la réunion d'un ensemble suffisant de données et d'objets.

Ces données collectives ont un statut particulier. Elles ont une existence temporaire, bornée par la publication. Elles ne confèrent pas à l'ayant droit une propriété ou un droit d'exploitation commerciale, mais un droit d'accès à des fins de recherche. Du reste, elles concernent surtout des données de recherche intermédiaires, des données non publiées ou des matériels de recherche, qui sont des inputs pour de futurs travaux ou pour des applications. Elles mêlent les caractères des biens privés et des biens publics : elles sont accessibles au sein du réseau, mais excluent les non-membres. En cela, elles confèrent un avantage particulier aux participants au projet, qui seront réticents à l'idée de les divulguer trop tôt dans l'espace public.

L'existence des données collectives renvoie à la croissance des nouvelles formes d'organisation de la recherche, en consortium ou en réseau coordonné. Le partage des données dans les réseaux scientifiques n'est toutefois pas une nouveauté (Kholer, 1994). Les scientifiques, académiques et industriels, utilisent couramment des réseaux d'échange fondés sur la réci- 
procité pour accéder à des matériels rares ou à des savoir-faire très spécifiques, qui ne sont pas disponibles sur le marché ou dans l'espace scientifique public (Cassier, 1995). Dans ce cadre, la valeur industrielle ou commerciale des données est suspendue (les données doivent être réservées à un usage exclusif de recherche, sous peine de rupture de l'accord). L'échange est fondé sur la disposition à donner et à recevoir et sur l'obligation de rendre. La nouveauté réside ici dans l'explicitation des règles de circulation, rendue nécessaire par la concurrence interfirmes au sein du consortium et par le renforcement des contraintes du marché sur les données. Le droit d'exclusion du réseau, en cas de refus de jouer le jeu de la réciprocité, reste toutefois tacite. Autre différence, dans les réseaux d'échanges scientifiques classiques, le contrôle s'effectue généralement dans le cadre d'échanges bilatéraux ( $A$ choisit $B$ pour réciproquer avec lui), dans des relations de proximité ou de connaissances interpersonnelles (la confiance et la réputation jouent un rôle majeur). Les consortia européens qui nous intéressent dessinent des espaces de collaborations multilatérales, beaucoup plus difficiles à contrôler, ce qui justifie également l'écriture des règles de circulation.

La troisième innovation des Guidelines est la plateforme industrielle, qui est un club d'utilisateurs industriels non directement membre du consortium, et qui acquitte un droit d'accès aux données produites par celui-ci. Elle constitue un espace semi-public ou semi-privé (tous les utilisateurs industriels intéressés peuvent y entrer). Le cas échéant, les membres de la plateforme pourront conclure des contrats avec les participants au consortium, sans toutefois que cela puisse leur conférer des droits sur les données du projet. Si des plateformes industrielles ont été créées à la périphérie de plusieurs consortia européens (réseau Levure, réseau Bacillus subtilis), cela n'a pas été possible dans le projet Lipases. Les industriels membres du consortium s'y opposèrent, de crainte de devoir partager les bénéfices de la coopération avec d'autres qui n'avaient pas engagé de ressources dans le projet. Il faut souligner le fait que les plateformes industrielles qui fonctionnent ont été créés à la périphérie de réseaux de laboratoires publics au sein desquels le conflit sur le partage de la rente est moins vif.

Enfin, ce système incite à une publication relativement rapide des résultats de recherches, ce qui satisfait les universitaires participant au projet. Si la publication des résultats peut être différée de quelques mois (11), les universitaires mettent en balance cette contrainte avec l'accélération de leurs travaux qui, grâce aux collaborations industrielles, bénéficient d'opportunités généralement non substituables par des collaborations avec des laboratoires publics. Les industriels n'ont pas intérêt non plus à trop

(11) Toutes les données ne passent pas nécessairement dans les trois cercles successifs (team, pool, public). Elles peuvent être versées directement dans le domaine public sur la décision de leur propriétaire [Rules and guidelines, article 7]. 
freiner les publications, afin d'attirer les universitaires les plus réputés. Ils publient eux-mêmes, pour bénéficier d'une rente associée à la publicité de leurs travaux et pour participer à la vie de la communauté scientifique.

\section{La circulation des matériels biologiques}

Si les règles de bonne conduite jouent un rôle important dans la régulation des échanges du consortium, il existe toutefois d'autres modalités de partage des ressources et des résultats de la recherche. Elles concernent notamment la circulation des matériels biologiques qui joue un rôle central dans les stratégies d'appropriation des acteurs. Ces règles de circulation des matériels sont le plus souvent tacites. Les compromis entre le privé, le collectif et le public sont ici particulièrement subtils (ils sont parfois inscrits dans les types de matériels utilisés).

La réunion d'une collection d'enzymes a été le premier souci du consortium. Le rassemblement d'une telle collection pose bien entendu des problèmes d'appropriation aigus dès lors que les enzymes appartiennent à des propriétaires différents et que certaines souches sont gardées strictement confidentielles dans les murs de l'entreprise. Comment gérer le dilemme entre appropriation privative et recherche collective? On observe tout d'abord une circulation très locale des enzymes, à l'intérieur de chaque sous-projet. On peut répertorier des échanges de matériel biologique entre les membres d'un sous-projet : par exemple, l'industriel du sous-projet 3 a reçu des souches sauvages en provenance de ses partenaires universitaires et leur a retourné des matériels produits par biologie moléculaire. Mais ces matériels ne sont pas communiqués aux autres groupes. L'appropriation passe ici par le contrôle étroit de la circulation des matériels aux partenaires directs, et non concurrents, de l'entreprise. On observe ensuite le rassemblement de la collection de toutes les souches d'enzymes, mais entre les seules mains d'un laboratoire public chargé de faire une recherche spécifique. Le laboratoire du CNRS de Marseille reçoit des échantillons de tous les participants, mais dans le cadre d'une circulation soigneusement cloisonnée. Lui-même ne doit pas les communiquer aux autres membres du consortium et devra les restituer à leurs propriétaires au terme de l'étude. Il représente un lieu neutre de la coopération. On observe enfin l'utilisation, par les entreprises, de matériels biologiques témoins qui sont communiqués au consortium, tandis que les souches les plus confidentielles qu'elles utilisent dans leurs procédés ou leurs produits restent dans leurs murs. Les souches témoins sont suffisamment proches des souches secrètes pour qu'il soit possible de récupérer des résultats transposables, et elles en sont suffisamment éloignées pour ne pas divulguer les secrets industriels. Ces matériels témoins permettent le passage, sans perte d'informations, du domaine privé au domaine public et vice versa. 
La circulation des mutants d'enzymes produits dans le cadre du consortium offre un autre exemple de compromis entre bien privatif et bien public. La coopération entre un laboratoire du CNRS et une grande firme de biotechnologie danoise a abouti à la production d'enzymes mutantes qui sont d'un grand intérêt industriel. Le laboratoire du CNRS travaille depuis plus de 20 ans sur une lipase du pancréas. Il a accumulé toute une collection de biomatériels et de données. Cette lipase a une propriété particulière, celle de dégrader à la fois les corps gras, comme les huiles, et des phospholipides, comme le jaune d'œuf. Les chercheurs du CNRs décident de profiter de l'opportunité de la collaboration pour faire établir la séquence génétique de l'enzyme. La société industrielle, fortement équipée en biologie moléculaire, séquence le matériel que lui envoie le CNRS. Les deux parties découvrent que cette enzyme est un mutant naturel. Elles décident de produire des mutants artificiels, à la fois pour approfondir les connaissances sur l'enzyme en question et pour les besoins de l'industrie des détergents. Comment faire circuler les mutants entre le laboratoire et l'industrie en évitant de divulguer des secrets industriels? Les partenaires s'entendent pour fabriquer deux types de mutants : des mutants de lipase pancréatique à partir des collections du laboratoire public, et des mutants de lipase d'origine microbienne à partir des souches privées de l'entreprise. $\mathrm{Si}$ l'entreprise fabrique les deux types de mutants, elle n'envoie au laboratoire que les mutants dérivés des collections académiques tandis qu'elle conserve dans ses murs ses propres mutants. La dichotomie entre «mutants publics» et «mutants privés» permet de préserver la propriété de l'entreprise qui bénéficie néanmoins des informations du laboratoire. En effet, elle reçoit les observations que le laboratoire effectue sur les mutants publics et les transfère immédiatement sur ses mutants privés, qui sont très proches des premiers: «On a l'information en même temps, et chacun l'utilise pour son usage. » [Directeur du laboratoire CNRS]. Le partage entre public et privé repose sur une circulation cloisonnée des matériels et sur la possibilité de transposer les informations des uns aux autres (des matériels témoins permettent, là aussi, le passage entre le public et le privé).

\section{L'invention de règles de propriété et de circulation des connaissances par les chercheurs : l'importance des régulations locales}

Tous les consortia européens de biotechnologie que nous avons étudiés (12) ont élaboré des consortium agreement qui complètent les contrats

(12) Outre le consortium sur les lipases, nous avons étudié cinq autres consortia, sur la levure, la bactérie Bacillus subtilis et le gène de prédisposition au cancer du sein.
Voir Cassier (1998), programme Colline, réalisé pour la Commission européenne, DG XII, Document IMRI, Université Paris Dauphine. 
types européens sur des points sensibles de la coordination, et notamment sur la question de la circulation des données et de l'attribution de la propriété des résultats de recherches. Il s'agit, en premier lieu, d'imaginer des solutions pour pallier l'absence de délai de grâce dans le droit des brevets en Europe, qui fait que le chercheur qui publie son invention avant de déposer une demande de brevet se voit opposer sa propre publication. Ceci à la différence du droit des brevets des États-Unis qui préserve les droits de propriété industrielle du chercheur qui a publié dans un délai d'une année. Les participants doivent donc veiller aux délais de divulgation de leurs résultats et prévoir des délais de publication qui préservent leurs droits de propriété industrielle ou les droits qu'ils pourront céder aux entreprises intéressées.

En second lieu, les participants s'efforcent parfois de limiter l'obligation de partage des données contenue dans le contrat type européen. Si les connaissances intermédiaires produites au cours du projet ainsi que les résultats finals sont la propriété de celui qui les a obtenus, ils doivent être accessibles à tous les participants du projet. Dans le contexte du consortium sur les lipases, qui associe des firmes largement concurrentes, celles-ci ont tenu à renforcer leur protection individuelle en créant des zones d'accès réservé (les cercles des différentes équipes). Ces jeux de règles s'efforcent enfin de compléter les dispositions des contrats européens en matière de coopération en organisant, de manière circonstanciée, les échanges entre les participants en donnant, par exemple, une existence aux pooled data ou données de réseau.

Ces arrangements locaux sont élaborés par les chercheurs eux-mêmes, le plus souvent à l'écart des services de propriété industrielle des firmes et des organismes publics de recherche. Dans le consortium sur les lipases, elles ont été discutées dans le cadre de réunions privées qui regroupaient, soit les cinq coordinateurs des équipes, soit les vingt-deux membres du réseau. L'écriture a été déléguée à deux universitaires qui ont été chargés de faire des propositions dans leurs domaines de recherche respectifs, la cristallographie et la cinétique (13). Lorsqu'il a été question de faire signer les Rules and guidelines du projet Lipases par les services juridiques des firmes, les participants ont préféré s'en tenir à un accord écrit mais non ratifié.

Le statut de ces règles est donc singulièrement complexe. Les Guidelines du consortium sur les Lipases s'apparentent à une règle de conduite entre scientifiques, analogue à celle qui régit les réseaux scientifiques informels. Toutefois, elles ont une inscription formelle et une certaine existence

(13) Ceci dans la mesure où la morphologie du réseau et les types de données produites n'y étaient pas les mêmes : il y a cinq sources productrices de données pour la cristallographie et une seulement pour la cinétique. Concernant les données cristallo- graphiques, il est également possible de les publier partiellement en gardant confidentielles certaines coordonnées atomiques. Les règles doivent être adaptées à la configuration du réseau et des données. 
juridique. Elles prennent la forme d'un contrat (elles comprennent un préambule, des définitions, des règles de divulgations des résultats, des instances de surveillance et d'arbitrage, des procédures en cas de litiges) et elles en ont également le sens (une autorité extérieure, administrative ou judiciaire, pourrait s'appuyer sur le document rédigé par les participants pour leur rappeler le sens de leurs engagements initiaux ou pour interpréter une requête particulière). Ces règles produites par les scientifiques prennent également appui sur les contrats types et les institutions communautaires, et par là se greffent sur la régulation existante. Elles s'inscrivent dans le cadre des contrats passés entre la CEE et les participants : «Le projet est composé de cinq équipes. Chaque équipe a conclu un contrat séparé avec la Commission des Communautés européennes. » [Article 3]. Elles s'appuyent sur l'institution des coordinateurs prévue par les contrats types. Elles reprennent les règles de confidentialité contenues dans les contrats : «Les participants sont liés par les règles de confidentialité de leur contrat. » [Articles 8 et 9]. L'autorité extérieure qu'elles désignent en cas de problèmes d'interprétation ou de litiges est le scientific officer de la Commission européenne responsable du contrat. Ce dernier a d'ailleurs veillé, tout au long de l'écriture des règles, à leur compatibilité avec le contrat type européen.

Ce processus de régulation locale est hybride entre les formes «d'autodiscipline du milieu professionnel» (Hermitte, 1988), qui aboutissent à des accords privés sous forme de codes de bonne conduite, et des formes juridiques. En élaborant des règles locales, les acteurs mobilisent et mettent en pratique les principes de coopération et de partage des données contenus dans les contrats types européens. Ils les adaptent à la configuration singulière des réseaux (qui sont susceptibles d'associer des laboratoires académiques et industriels ou encore de mettre ensemble des participants concurrents). Elles permettent, plus largement, de compléter les règles de propriété industrielle existantes : elles pallient l'absence de délai de grâce en Europe, permettent de protéger des connaissances de base non brevetables avec le droit de premier usage des connaissances, ou encore de stimuler le partage des données dans un réseau sans détruire toute protection individuelle. Dans la mesure où elles sont produites et mises en œuvre directement par les acteurs locaux, elles favorisent une coordination rapide, en évitant des retours trop longs devant les juristes qui pourraient demander des garanties supplémentaires. Toutefois, ces solutions locales et ad hoc peuvent aboutir à de grandes disparités, par exemple pour ce qui concerne les délais de divulgation des données. On observe ainsi, selon les consortia, des règles de divulgation immédiate ou différée, des solutions de publication complète ou partielle, des délais de confidentialité qui varient de un à douze mois.

Dans quelle mesure faut-il harmoniser ces différents jeux de règles, voire les généraliser? Tout d'abord, ces Guidelines circulent entre les réseaux scientifiques européens : les chercheurs du réseau Lipases ont «copié » les règles du Perfect gentleman sequencer élaboré par les chercheurs du 
réseau européen sur la levure en 1989. Ceux du réseau Bacillus subtilis ont également repris dans leur contrat des dispositions du réseau Levure. La diffusion des «bonnes pratiques», par exemple des solutions de diffusion concentrique des données, permet d'en tester la robustesse. On peut également les généraliser en les intégrant au contrat type de la CEE (14). Plus largement, on peut estimer que ces règles locales représentent du «droit en train de se faire», à savoir le nouveau droit de la propriété intellectuelle dans un contexte d'invention collective. Les nouvelles solutions expérimentées (droit de premier usage des connaissances, pooled data, plateformes industrielles, etc.) étant susceptibles de se généraliser (15). Les scientific officer de la Commission européenne et les scientifiques qui assurent la coordination des projets engrangent des solutions qu'ils peuvent faire partager à leurs collègues. Dans le même temps, les échanges de bonnes pratiques ne doivent pas conduire à une convergence trop rapide et trop poussée des solutions en matière de gestion des connaissances, sous peine de réduire les possibilités de négociation de compromis locaux originaux.

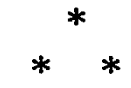

L'analyse de la gestion des connaissances dans un consortium de recherche mixte, réunissant des laboratoires académiques et des firmes industrielles, permet en premier lieu de révéler la capacité d'invention des chercheurs en matière de régulation économique et juridique. Dans le cas étudié, les chercheurs ont court-circuité les services juridiques des firmes et des institutions scientifiques. Dans d'autres cas, ils imaginent des jeux de règles qu'ils font ensuite relire par leurs services de propriété industrielle. Ce faisant, comme l'avait envisagé Callon (1986), les scientifiques inventent des faits de société en même temps que des faits techniques. On voit également émerger dans ces nouvelles formes d'invention collective des normes de diffusion de la science fondées sur des compromis locaux entre protection individuelle, partage des données dans un réseau et versement dans l'espace public. En fait, les chercheurs gèrent couram-

(14) Les rédacteurs du Perfect gentleman sequencer souhaitaient que certaines des dispositions qu'ils prévoyaient, par exemple sur les processus de contrôle de la qualité des données, soient intégrées aux contrats de la CEE lors du programme de biotechnologie suivant : «Le présent document n'a pas de caractère contractuel pour le programme Bridge sur les chromosomes II et IX. Il est toutefois proposé de mettre en œuvre immédiatement les règles sur la distribution des tâches et de la propriété et de mettre en œuvre plus graduellement les règles de contrôle de la qualité qui pourraient devenir pleinement contractuelles dans le prochain programme biotechnologique. »

(15) Dans un domaine voisin, celui de l'obtention végétale, M. A. Hermitte (1988) a montré la génèse progressive d'un nouveau droit, le droit de l'obtention végétale, dans le cadre de contrats et d'associations créés par les professionnels. 
ment plusieurs degrés de confidentialité de leurs données, depuis la confidentialité des données de laboratoire en cours de validation ou qui ont un intérêt industriel potentiel, à la divulgation de données dans des réseaux de partenaires proches, et à la publication dans des revues scientifiques. Une telle conclusion conduit à circonscrire les normes mertoniennes de la science ouverte (à l'encontre des présupposés de Dasgupta et David, 1994) et à privilégier l'hétérogénéité des manières de faire de la science et de gérer les connaissances (Pestre, 1997). Pour Merton (1942), la science a un statut de propriété commune. Les scientifiques bénéficient d'une sorte de propriété intellectuelle sous forme de priorité de la découverte validée par leurs pairs, mais il ne s'agit en aucun cas d'une appropriation exclusive. Dans la mesure où la science fait partie du domaine public, la communication pleine et entière des résultats est une règle impérative : «Le secret est l'antithèse de cette norme.» Si Merton relève que des scientifiques comme Einstein ou Millikan ont été conduits à prendre des brevets, notamment pour préserver le libre usage de leur travail, il proclame que le statut de bien commun de la science est incompatible avec la définition de la technologie comme propriété privée dans l'économie capitaliste. Nous montrons, pour notre part, que les chercheurs sont engagés dans plusieurs modes de gestion des connaissances, notamment à l'interface de la science et du marché, qu'ils sont susceptibles d'imaginer et de négocier une grande variété d'arrangements locaux dont les règles sont éventuellement transposables et généralisables, et que l'hypothèse d'une culture générale de la science à laquelle les chercheurs doivent se conformer (à moins qu'ils n'en dévient) est fortement réductrice.

L'examen des solutions retenues par le consortium sur les lipases et les consortia européens voisins laisse entrevoir des compromis originaux entre ce qui relève du domaine privé et du domaine public. On rejoint ici une conclusion de l'économiste K. J. Arrow, qui écrivait en 1994: «Nous allons rencontrer des nouvelles formes de propriété qui comportent à la fois des aspects de bien public et de bien privé.»Il en va ainsi des «données collectives» que nous appelons aussi données de réseau, qui désignent des entités partagées dans le consortium le temps de la recherche afin de stimuler l'invention collective des participants. Il ne s'agit pas d'une propriété collective des participants, à l'instar d'un brevet détenu en copropriété, mais d'un droit d'accès partagé aux connaissances afin d'élaborer de nouveaux résultats. Ces données collectives sont partagées au sein d'un réseau, mais sont protégées vis-à-vis de l'extérieur. En cela, elles diffèrent des données publiques qui sont accessibles à tout utilisateur. En effet, si l'organisation du réseau a pour but de stimuler l'invention collective grâce à la mise en commun des données de recherche, elle vise aussi un meilleur contrôle des transferts de connaissances entre les acteurs. On peut voir ainsi proliférer des alliances semi-privées qui retiennent les connaissances dans des espaces coopératifs étroits, dominés par quelques acteurs privilégiés. Le degré d'ouverture des consortia de recherche visà-vis des espaces publics de circulation des connaissances (banques de don- 
nées publiques, revues) est une question cruciale pour la politique scientifique. On mesure l'importance de suivre les nouveaux partages qui sont établis entre le domaine public et privé de la recherche (Hilgartner, 1995), de collecter et d'analyser des types de solutions imaginées par les acteurs locaux (accès immédiat ou différé aux données, divulgation partielle versus divulgation complète, cercles d'accès plus ou moins larges, etc), d'envisager la génèse et la diffusion de nouveaux droits de gestion des connaissances.

\section{Maurice CASSIER}

Centre de Recherche Médecine, Sciences, Santé et Société (CERMES) - CNRS

182, boulevard de la Villette, 75019 Paris

cassier@est.jussieu.fr

\section{RÉFÉRENCES BIBLIOGRAPHIQUES}

Aldrich H. E., Sasaki T., 1995. - «R \& D consortia in the United States and Japan », Research Policy, 24, pp. 301-316.

Arrow K. J., 1994. - «Methodological individualism and social knowledge», American economic review, May, pp. 1-9.

Callon M., 1986. - «Éléments pour une sociologie de la traduction. La domestication des coquilles Saint-Jacques et des marins-pêcheurs dans la baie de Saint-Brieuc ", L'Année sociologique, 36, pp. 169-208.

- 1992. - «Sociologie des sciences et économie du changement technique : l'irrésistible montée des réseaux technico-économiques», dans Ces réseaux que la raison ignore, Paris, L'Harmattan.

Callon M., Larédo P., Mustar P., 1995. - «Réseaux technico-économiques et analyse des effets structuraux», dans La gestion stratégique de la recherche et de la technologie: l'évaluation des programmes, Paris, Économica, pp. 415-462.

Cassier M., 1995. - Les contrats de recherche entre l'université et l'industrie : l'émergence d'une nouvelle forme d'organisation industrielle. Thèse de socio-économie, Paris, École des Mines.

- 1996. - «Les contrats de recherche entre l'université et l'industrie : les arrangements pour la production de biens privés, de biens collectifs et de biens publics », Sociologie du travail, 38, 3, pp. 377-390.

Cohendet P., Héraud J.-A., Zuscovitch E., 1992. - «Apprentissage technologique, réseaux économiques et appropriabilité des innovations», dans D. Foray, C. Freeman (éds.), Technologie et richesses des nations, Paris, Économica, pp. 63-78.

Dasgupta P., David P., 1994. - «Toward a new economics of science», Research policy, 23, pp. 487-521.

Grindley P., Mowery D., Silverman B., 1994. - «SEMATECH and collaborative research : lessons for the design of high-technology consortia», Journal of policy analysis and management, 35, 4, pp. 723-758.

Hermitte M. A., 1988. - «Histoires juridiques extravagantes : la reproduction végétale », dans L'homme, la nature, le droit, Paris, Christian Bourgeois, pp. 40-98.

- 1995. - «Le concept d'économie de la connaissance à l'épreuve du droit » [Projet de recherche communiqué par l'auteur, 12 pages]. 
Revue française de sociologie

Hilgartner S., 1995. - Data access policy in genome research, Ithaca, Cornell University Press.

Kohler, 1994. - The lords of the fly, Chicago, University of Chicago Press.

Merton R. K., [1942] 1973. - «The normative structure of science», dans N. W. Storer (ed.), The sociology of science : theoretical and empirical investigations, Chicago, University of Chicago Press.

Pestre D., 1997. - «La production des savoirs entre académie et marché. Une relecture historique du livre "The new production of knowledge" édité par M. Gibbons", Revue d'économie industrielle, 79, pp. 163-172 [Numéro spécial : L'économie industrielle de la science]. 